\title{
Agôn
}

Revue des arts de la scène

$6 \mid 2013$

La Reprise

\section{Le fantôme, le monument et le combustible}

Introduction du dossier sur la reprise dans les arts de la scène

Alice Carré, Marion Rhéty et Ariane Zaytzeff

\section{(2) OpenEdition \\ Journals}

Édition électronique

URL : http://journals.openedition.org/agon/2806

DOI : 10.4000/agon.2806

ISSN : 1961-8581

Éditeur

Association Agôn

Référence électronique

Alice Carré, Marion Rhéty et Ariane Zaytzeff, « Le fantôme, le monument et le combustible », Agôn [En ligne], 6 | 2013, mis en ligne le 21 février 2014, consulté le 04 mai 2019. URL : http://

journals.openedition.org/agon/2806 ; DOI : 10.4000/agon.2806

Ce document a été généré automatiquement le 4 mai 2019.

Association Agôn et les auteurs des articles 


\section{Le fantôme, le monument et le combustible}

Introduction du dossier sur la reprise dans les arts de la scène

Alice Carré, Marion Rhéty et Ariane Zaytzeff

« Tout est dit, et l'on vient trop tard depuis plus de

sept mille ans qu'il y a des hommes qui pensent ${ }^{1}$.»

Jean de La Bruyère

\section{Détricoter les fils de la reprise : entre les vides et les pleins}

1 La scène aura marqué les esprits: Véronique Doisneau, seule sur le vaste plateau, rejouant un extrait du deuxième acte du Lac des cygnes dans la chorégraphie de Noureev, dans le spectacle de Jérome Bel qui porte son nom² ${ }^{2}$ Elle est à dix jours de sa retraite de danseuse de l'opéra de Paris. Seule représentante du corps de ballet, sans les décors, sans les étoiles, sans les costumes, elle demande au régisseur la bande son correspondant à l'extrait et danse le vide. C'est bien dans son dénuement que la partition apparaît, les longues pauses, de dos, l'attente du repère musical, pour un pas et de nouveau une longue pause. Elle dit sa souffrance à danser ce rôle. Elle dit qu'elle avait envie de crier. Elle danse ce qu'elle a dansé tant de fois et que pourtant personne n'avait vu. Conçue comme un assemblage de plusieurs extraits que Véronique Doisneau a dansés pour l'Opéra de Paris, cette pièce de Jérôme Bel fait le vide autour de la danseuse du corps de ballet, ne garde que ce fragment, ne reprend que sa partition à elle, par là, son histoire d'interprète. Ce vide immense, le remplir à nouveau, autrement. Par la lumière sur sa présence. Elle était bien là sur scène, Véronique Doisneau, il ne fallait pas l'oublier. Elle monte à nouveau sur scène pour le dire.

2 Poser la question de la reprise dans les arts de la scène, c'est savoir qu'il faudra cheminer entre les vides et les pleins des archives et de la mémoire, plus précisément par les vides et par les pleins. Combler les trous, vider pour rendre plus plein, faire apparaître les 
oublis, quelle que soit la formule, c'est à cet endroit que la reprise noue son fil. «Tout est dit, et l'on vient trop tard », dit La Bruyère. Alors on reprend.

3 Cette problématique traverse la reprise. S'il relève de la gageure de l'écrire au singulier, tant les formes, la nature, les intentions, les dramaturgies des reprises diffèrent, tentons de situer ces formes selon une échelle qui irait de la plus fidèle au spectacle préexistant en termes d'écriture, à la plus fidèle au mouvement créateur du spectacle :

- La reprise ou remontage: par l'équipe de la création, parfois en raison du succès ou d'opportunités nouvelles de diffusion, le spectacle ne change que par l'adaptation nécessaire à quelques paramètres (changement d'interprète, changement de lieu etc.) ; ces paramètres sont de plus en plus nombreux selon le temps qui s'est écoulé entre la création et la reprise, jusqu'aux reprises après la mort de son créateur.

- La reconstitution et la reconstruction : une recherche historique s'impose, à travers laquelle on cherche à remplir les vides du temps ou de l'oubli. La reconstitution est le fantasme du plein, jusqu'au reenactment de scènes historiques (la bataille de Waterloo, ses costumes, ses mousquets et ses chevaux).

- La recréation : la pièce nouvellement créée ne cherche pas à imiter la première version et ne s'encombre pas de l'utopie de retrouver le même, elle traverse, librement, une intention initiale de l'œuvre3 .

- L'emprunt, la citation, le recyclage: prenant de multiples noms selon les créateurs, elle s'intéresse moins à l'objet qu'elle reprend qu'à sa transformation ${ }^{4}$, la fidélité prenant en compte les vides de l'original pour faire advenir de nouveau.

Si certains la brandissent comme une résistance au renouvellement permanent, à l'exigence d'innovation, à l'obsolescence des formes éphémères du spectacle vivant, d'autres voient au contraire la reprise comme une façon de remplir les salles en temps de crise, et d'autres encore, loin de céder au défaitisme de La Bruyère, la considèrent comme nécessaire, évidente, une façon constructive de créer en prenant en compte ce qui a déjà été fait. En dehors de certaines tentatives nostalgiques d'accéder à un passé dont on refuse la perte, tous invitent à dépasser le paradigme du nouveau et de l'ancien, afin de créer entre l'histoire et présent des dialogues plus fertiles.

Que l'on parte de la définition la plus pragmatique - la définition économique du mot s'appuie, selon Daniel Urrutiaguer sur un " périmètre temporel très variable », mais s'applique à propos d'un spectacle dont la tournée a cessé depuis au moins une saison ${ }^{5}$ ou de la plus libre - avec des pratiques artistiques qui reprennent sur le mode de la citation, de l'allusion... - la reprise est définie par rapport à ce qui précède. C'est de là qu'elle tire à la fois son identité (elle se définit par rapport à, en pour, en contre, ou en ailleurs), son processus de création (utilisation, transmission, appropriation de matériaux ou documents préexistants), la quasi-garantie d'un marquage du temps (inscription de l'artiste dans une lignée, légitimation d'une démarche artistique par rapport aux pairs), sa valeur dans un système de consommation culturelle (garantie d'un emballage marketing et d'une couverture médiatique).

\section{Ce qui précède}

6 Quel est donc ce qui précède? Une œuvre, le souvenir d'une œuvre, le souvenir d'une expérience d'une œuvre? Un interprète, un interprète ayant vieilli, un groupe 
d'interprètes? Une partition, un document, des notes, des traces? Plus on s'en approche, plus il semble échapper : que reprend-on quand on reprend?

7 On conclurait trop vite que tout se vaut, que la reprise existe dans tous ses états, partout et tout le temps. Sans nier cet écueil et la difficulté à cerner le sujet, parler de la reprise, c'est parler de la création, de ce bégaiement $d u$ « re ». Cette propension à repasser par les mêmes chemins, à battre une terre battue par d'autres, ne masque pas le trépidant de la découverte ni l'envie de la surprise. Il y a quelque chose du plaisir d'aller fouiller dans le grenier, sachant pour une part ce qu'on risque d'y trouver, prenant le risque de n'y justement rien trouver. Qu'on s'y amuse un moment, les yeux ébahis devant la caverne d'Ali Baba, qu'on soit atrophié, asphyxié par tout ce qui s'y trouve, qu'on y mette le feu une bonne fois pour toute... au final, ce qui nous intéresse, c'est cette envie d'ouvrir la porte et de quelle manière on l'ouvre.

8 Pour définir ce rapport à ce qui précède, prenons l'exemple de la reconstitution, forme paradoxale de la reprise dans des arts de la scène où la composition, l'entretien du grenier et la transmission des clés se font majoritairement de façon directe, de personne à personne. Quand les reprises interviennent après la mort des artistes qui ont créé les œuvres, elles mettent alors en jeu certaines pratiques de reconstitution. Ainsi Millicent Hodson et Kenneth Archer, une chorégraphe et notatrice et un historien d'art, proposent avec le Joffrey Ballet en 1987 à Los Angeles une version du mythique Sacre du printemps de Nijinski et Stravinski, disparu des scènes après une première reprise de Massine en $1920^{6}$. Leur travail, qui fera le tour des scènes internationales, s'apparente à de la "reconstitution ", autour d'un travail d'archives à partir de dessins de Valentine Hugo, de notes d'une assistante de Nijinski, de quelques souvenirs de spectateurs et de critiques. Soulignant la part de fantasme qui opère nécessairement dans chaque "reconstitution " et l'impossible quête que représente la partition de Nijinski, Dominique Brun ${ }^{7}$, qui prépare actuellement une nouvelle reconstitution du ballet de 1913, empruntait à Frédéric Pouillaude sa typologie autour de trois rapports différents à l'œuvre d'origine : la version, l'œuvre archive et l'œuvre signe ${ }^{8}$. La version emprunte le cadre d'un projet précédent en restant dans une relative indifférence pour l'œuvre initiale, l'attention étant portée sur l'objet à venir, la création nouvelle ; l'œeuvre archive cultive le fantasme de revenir à l'œuvre d'origine, en tentant de s'appuyer sur les traces qui en sont conservées ; l'œuvre signe s'appuie sur l'identité d'une œuvre, sans avoir l'ambition d'en retrouver l'essence. Ces trois rapports à l'œuvre chorégraphique indiquent trois attitudes face à l'œuvre source qu'empruntent les artistes contemporains : le respect et la mystification de l'objet disparu, la réutilisation du matériau historique comme source de l'œuvre contemporaine, l'utilisation libre et allusive de l'œuvre d'origine comme une citation ou un clin d'œil au sein d'un questionnement personnel.

9 Il y a donc au sein des pratiques de la reprise une double tension entre le passé et le présent, illustrée par Kierkegaard quand il distingue reprise et ressouvenir : «Reprise et ressouvenir sont un même mouvement, mais en direction opposée; car, ce dont on a ressouvenir, a été : c'est une reprise en arrière ; alors que la reprise proprement dite est un ressouvenir en avant ${ }^{9}$. »

10 Ainsi dans les pratiques visant à la réitération, à la reproduction du même objet et à la célébration du passé se trouvent ce que les nord-américains nomment « reenactment » ou "re-enactment», reconstitution d'événements historiques afin d'en explorer les impensés et d'en proposer des interprétations nouvelles et de réparer des oublis. Ce type de spectacle, ayant abouti à de nombreuses reconstitutions de batailles de la guerre de 
sécession américaine, à celle, annuelle en Angleterre de la bataille de Waterloo ${ }^{10}$, a parfois pour but selon l'analyse qu'en fait Slavoj Zizek ${ }^{11}$ de figer le passé, de rendre inoffensif le moment de surgissement et de le réintégrer de manière continue à notre présent. Ainsi, celui-ci prend-il l'exemple de la prise du palais d'hiver de Leningrad, moment crucial de la Révolution russe: repris trois ans après l'événement historique de 1917 à SaintPétersbourg, le reenactment réunissait 6000 personnes - ouvriers, soldats et marins - et s'adressait à 150000 spectateurs, soit un quart de la population de la ville à l'époque. Au moment même du siège de Leningrad en 1920, Staline signifiait au peuple russe, par cette pratique artistique de masse, que cette révolution n'était pas un simple coup d'état porté par un petit groupe de bolchéviques, mais constituait un véritable événement ayant déclenché une émancipation sociale sans précédent. Ainsi, la ré-exploration en acte du passé est un « ressouvenir en avant » qui, en écrivant une mémoire, définit le présent au service d'une idéologie et d'un pouvoir politique.

\section{Portrait de l'artiste en copieur}

11 Si ce qui précède est ce par rapport à quoi se définit la reprise, rapport qui déploie l'étendu des possibles positionnements, de la part des artistes mettant en scène ces reprises comme de la part de ceux qui les reçoivent, il y a l'idée sous-jacente que ce qui précède, c'est l'original. Dès lors, la reprise serait une forme de copie $^{12}$. Si la copie présuppose de l'entité, de l'existence d'un original - et l'on a vu que pour le spectacle vivant, cela pose question de façon intrinsèque, par sa nature mouvante - l'intérêt réside dans ce qu'elle met en tension aux deux étendues du spectre : la copie comme fantasme d'atteindre l'original d'un côté, la copie comme moyen de ne pas idéaliser l'original, parfois associé à un refus d'ajouter de la valeur en permanence et à un refus de la notion téléologique de progrès. Il y a plaisir simple à refaire, à ce "encore». Freud nommait comme « un des souhaits les plus fervents de l'humanité » la possibilité « d'expérimenter quelque chose deux fois $»^{13}$. Si Freud écrit ceci à propos de l'hypnose, Inke Arms, dans son texte History will repeat itself ${ }^{14}$, émet l'hypothèse que c'est le même fantasme qui se joue pour le reenactment, c'est-à-dire que cela permet au protagoniste, en rejouant une scène déjà vécue, de le faire en pleine conscience : étant au centre, il acquiert la possibilité de prendre de la distance.

12 Aux Etats-Unis les performance studies, par le biais de cas d'étude américains et l'interdisciplinarité des cadres d'analyse, développent une vision de la performance intrinsèquement liée à la reprise. La performance n'est alors plus seulement une forme d'art mais aussi un cadre théorique qui permet d'analyser les comportements et actions humains comme des performances. L'un des concepts fondateurs des performance studies, développé par Richard Schechner, est celui de "restored behavior", qui voit la performance comme un comportement restauré, c'est-à-dire un assemblage de comportements précédents. Pour Schechner, «la performance signifie : jamais pour la première fois ${ }^{15}$. » Dans cette théorie, le comportement n'est pas propre à un individu ou attaché à une personne, mais "séparé de ceux qui se comportent " et peut donc être " gardé, transmis, manipulé, transformé $»^{16}$. Dans sa réflexion sur le reenactment, Rebecca Schneider écrit que « toute pratique de la représentation, et en effet tout comportement de communication, est composé de réitération, engagé dans la citation, est déjà une pratique du reenactment, ou ce que Richard Schechner a appelé le «comportement

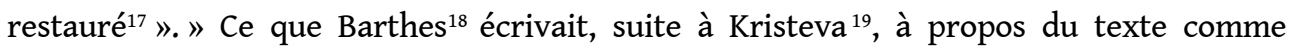


réagencement de citations, de fragments de textes précédents, socle à la notion d'intertextualitée ${ }^{20}$, Schechner l'étend à la sphère des comportements sociaux.

Mais si Barthes développe un cadre d'analyse pour l'étude des procédés d'écriture, tout à fait valable dans les arts de la scène, dès lors qu'on inclut dans cette écriture celle du plateau, des corps, des voix, des décors, de la lumière, des costumes, du son - chacune dans leur unité mais aussi dans leur assemblage - Schechner théorise à l'endroit du frottement entre ces écritures et le monde, c'est-à-dire dire du performatif. Concept majeur des performance studies, le performatif est le terme par lequel J. L. Austin, philosophe linguistique, présente le langage comme un acte qui ne sert pas à décrire mais à faire. Le performatif repose sur la citation et la réitération pour être intelligible et efficace, et étend la notion de langage à la communication non verbale. Ainsi, les concepts de comportement restauré et de performatif soulignent que toute performance fait appel à une histoire, un avant, allant jusqu'à demander avec Jacques Derrida si «tout n'a [...] pas commencé par la reproduction ${ }^{21}$ ».

Ce portrait de l'artiste en copieur ne saurait donc être complet sans la prise en compte de la réception, pierre angulaire de ce triangle entre ce qui lui précède, ce qu'il agit ou ce qu'il produit et ce qui est perçu. Dès lors, il s'agit moins de s'intéresser à la copie qu'à l'écart, non tant pour analyser le degré de distance à un original fantasmé, que pour interroger cet écart dans ce qu'il dit d'un rapport à l'histoire.

\section{Ne faut-il plus tuer le père?}

\section{L'archéologue, le collectionneur, le curateur et le biographe}

L'époque est à l'hypertrophie de la mémoire et à sa consommation ${ }^{22}$. Les arts de la scène n'y échappent pas : que l'on pense à la façon d'interpeller le document en scène, dans le processus de création ou au plateau; que l'on pense à la préoccupation pour la notation et la conservation en danse ; à la pratique du recyclage et du recours à la seconde main (objets, techniques) ; que l'on pense aux enjeux de légitimité qui façonnent un paysage et ses formes, dans la danse et plus récemment dans le cirque ; que l'on pense à l'outil médiatique qui sert à emballer nombre de produits culturels. Poser la question de la reprise, c'est choisir d'interroger les arts de la scène à l'endroit où ils sont aux prises avec l'histoire, c'est-à-dire avec le présent. La reprise est une seconde digestion et on ne cesse pas de mâchonner. Mais dans cette prise avec le temps, la question du «comment?» domine la question du "pourquoi? ?. Et de même qu'à la question "que reprend-on quand on reprend ?", la réponse pointe du doigt ce "comment », c'est-à-dire l'activité créatrice elle-même, plus que le redoublement de l'œuvre, cette prise avec le temps manifeste un intérêt pour les pratiques de l'historien, d'une part, pour la mémoire d'autre part, plus que pour l'histoire elle-même - ce qui n'est pas sans poser question, on y reviendra plus loin.

On distinguera quatre figures que se plaisent à emprunter les artistes s'essayant à la reprise : l'archéologue, le collectionneur, le curateur, le biographe. Réjouissons-nous, Foucault est passé par là, et son Archéologie du savoir (1969) aura construit la prise en compte du document comme monument, nécessitant la prise en compte de ses failles et des fils qui le sous-tendent pour le penser ${ }^{23}$. L'historien d'art Arnauld Pierre parle d'« archéomodernistes ", désignant par là la démarche de plasticiens qui « approchent la modernité par son archéologie et embarquent dans le moderne non seulement ce que 
l'histoire en a retenu, mais aussi ce qui n'a pas eu d'avenir, ce qui n'a pas débouché24 ». On pense par exemple au travail d'olga De Soto sur la Table verte de Kurt Jooss, mais aussi à Dominique Brun, à l'entreprise des Carnets Bagouet et du Quatuor Knust, du théâtre documentaire, à ces formes qui s'appuient sur la collecte de documents, de témoignages... Une autre figure serait celle du collectionneur. Franck Apertet ${ }^{25}$ estime que la reprise est aux arts vivant ce que la collection est aux arts plastiques. Si l'on y trouvera les grands événements et les pépites rares, c'est ici aussi que l'on pourrait classer les spectacles anniversaire en forme de compilation, comme le best-of Panorama Decouflé (2012, reprise en 2014), Spiegel de Wim Vandekeybus (2008), Impromptu de tg STAN (2009)... - entreprises qui répondent de diverses intentions festives, joyeuses, conservatrices ou commerciales. Mais la figure du collectionneur se double de celle du curateur, autre forme de manœuvre dans l'agencement de ces bouts du passé sur l'étagère. L'artiste se fait curateur de sa propre histoire, ou de son propre champ - on pense ici à Xavier Le Roy avec Rétrospective, à Boris Charmatz, Jérome Bel, aux gens d'Uterpan, Marina Abramović... La troisième figure serait celle du biographe ou de l'autobiographe, quelque part entre les deux, s'agissant souvent d'exprimer un «nous» plus qu'un «je». Isabelle Launay parle de «s'entredanser », comme Montaigne dans les Essais parle de «s'entregloser » et constate que si «la danse, comme tous les arts, s'invente dans une relation avec le monde, elle s'invente tout autant dans une relation avec elle-même ${ }^{26}$ ».

Ces figures font ressortir la construction ou du moins la conscientisation d'un rapport à l'histoire, compris comme prise avec le présent. Ne nous méprenons pas, la contemporanéité a toujours existé mais différemment selon les époques ${ }^{27}$, il s'agit de réinventer un rapport au passé.

\section{« Exit 30's, Enter 60's »}

«La question que je voudrais vous poser et à tous ceux qui sont plus jeunes que moi est : pourquoi venez-vous me parler aujourd'hui ? (...) Bien sûr, pour des questions d'égo, je me réjouis de vous parler. Mais historiquement, structurellement, que s'est-il passé28?»

Richard Schechner, posant la question de la reprise des avant-gardes, s'étonne de la nostalgie qui semble dominer les jeunes générations, questionnant cette position de respect pour le passé. « Exit 30's, Enter 60's ", tel était le titre d'un article écrit à l'époque ${ }^{29}$, afin de signifier que, si la scène et les arts des années 30 avaient été très marquants pour les États-Unis, la génération des années 60 devait prendre la place, tourner la page et s'en détacher. Cette posture d'irrespect, reprenant le constat de Pasolini sur la nécessité pour une génération de tuer ses pères pour exister historiquement ${ }^{30}$, semble se retourner aujourd'hui en son contraire, une forme de déférence par rapport aux générations précédentes. Ne faut-il donc plus tuer le père?

19 La question se pose avec une force particulière dans le cas des reprises. Ainsi Schechner, dont le spectacle Dionysus in 69, forme de contestation à l'égard des codes et des convenances de l'époque a été repris par diverses compagnies, s'étonne de la reprise quasi-religieuse qui était faite de la performance du Performance group. Comment expliquer que l'on reprenne ces formes contestataires et iconoclastes quand bien même ce qui semble être le propre de la performance est d'être vouée à la disparition pour n'exister que dans le présent ${ }^{31}$ ? Si l'essence même de la performance est le refus de la représentation et l'unicité du geste artistique, reprendre semble contradictoire. La question de la conservation du performance art est d'ailleurs au cœur de nombreux 
débats, opposant ceux qui refusent la reprise et re-présentation et ceux qui la défendent et l'explorent ${ }^{32}$.

La nostalgie qui touche les jeunes générations se focalise plus précisément sur la période contestataire et iconoclaste des années 60-70. Si on peut expliquer une partie de ce phénomène par l'absence de transmission par la génération de mai 68 de l'idée d'utopie, de révolte ou de refus de l'ordre établi et de leur mise en échec, il semble que la suivante ait une vision du monde plus désabusée, comme si « l'échec » de mai 68 avait relégué la contestation au statut d'action vaine. Par là même, il semble que la fascination pour ces performances subversives, brisant des tabous soit plus présente encore. L'attachement de nos sociétés postmodernes à cette époque se traduit en se saisissant de sa seule esthétique (de sa syntaxe, de son geste), seul élément tangible, à défaut d'être en capacité d'exprimer son rôle positif dans l'histoire. En découle alors une incapacité à la perte et à l'oubli, la tension entre l'exigence du souvenir et la nécessité de l'oubli ne trouvant pas de point de résolution. Cette nostalgie, cette mélancolie - par la suppression de la projection vers une délivrance - en est alors une des manifestations.

\section{Patrimoine et archive vivante}

21 Au-delà de la question des utopies et de la nostalgie pour la transgression propre à ces deux décennies, il semble que le lien nouveau au passé s'inscrive de façon plus générale dans une forme d'attention particulière portée au patrimoine. Le changement du rapport entre histoire et mémoire rendu nécessaire par les conséquences durables de la seconde guerre mondiale sur les sociétés devant se réinventer a ouvert pour la culture dans son sens large la question de sa conservation, par là, de sa possible transmission.

Depuis 1980, baptisée sous Giscard d'Estaing "année du patrimoine », s'est engagée la course au patrimoine, s'accompagnant de la déclinaison de toute une gamme de nouveaux monuments historiques ${ }^{33}$. La catégorie s'est considérablement élargie, voyant naître de nouvelles catégories de patrimoines protégés par l'UNESCO : patrimoine culturel, naturel, mais surtout "patrimoine immatériel de l'humanité ${ }^{34}$ ", visant à protéger et à conserver des formes d'art vivantes de type rituelles ou folkloriques. Ainsi, les formes rituelles, spectaculaires et folkloriques eurent leur label et mirent un pied dans le domaine de la conservation. Un phénomène de patrimonialisation de la culture semble, ces dernières décennies, toucher de plein fouet les arts vivants. L'une des manifestations de cette tendance est la création de liens étroits entre le musée et les arts vivants. Le musée de la danse, dirigé par Boris Charmatz à Rennes s'inscrit dans cette pensée de la conservation d'un répertoire et de pratiques de la danse, tout en cherchant à rendre cette mémoire vivante, à trouver, dans la contradiction entre mouvement et fixité, des formes nouvelles pour penser la danse. Il est également de plus en plus fréquent que l'artiste vienne s'approprier l'espace du musée pour y « exposer » la danse. Ainsi, plusieurs chorégraphes font l'exercice du transfert de l'espace de la représentation à celui de l'exposition, à l'instar de Damien Jalet au Louvre ${ }^{35}$, ou de Xavier Le Roy à la Fondation Tapiès de Barcelone, au musée de la danse ou au Centre Georges Pompidou ${ }^{36}$. Si la pratique de la reprise d'une œuvre chorégraphique existe depuis longtemps, la danse cherche aujourd'hui à se faire mémoire de sa propre histoire et à questionner son répertoire, à le reparcourir et le retracer.

D'autres disciplines ayant cherché plus récemment à se détacher de l'étiquette de " genre mineur », de "populaire», ou de «divertissement » font de la reprise l'endroit d'une 
recherche de légitimation nouvelle. Le cirque contemporain, qui, depuis les années 80 a cherché à questionner les notions d'écriture et de dramaturgie autour d'un alliage des formes et d'une rupture de la forme séquencée en numéro, réfléchit aujourd'hui à la constitution de son répertoire. Marie Fonte, dans l'entretien ${ }^{37}$ mené par Aurélie Coulon pour ce dossier, développe l'idée que reprendre un spectacle, c'est en fait poser la question de l'écriture et de la forme. La question de l'absence de répertoire en cirque oriente le travaille de la Compagnie Yoann Bourgeois et a motivé la reprise de Fugues ${ }^{38}$. De même, la question de l'écriture s'est posée pour Archaos, pour In vitro ${ }^{39}$ qui avait fait l'objet d'un "scénario, écrit au préalable à toute recherche physique et matérielle ${ }^{40}$ », qui a permis deux reprises du spectacle en 2009 : l'une avec les élèves de l'École de cirque de Rio cherchait à reprendre à l'identique la version de 1999, la seconde, recréée par les artistes d'Archaos, cherchait à s'émanciper de la première version pour en proposer une nouvelle. L'édition du scénario, exemple rare d'une écriture pour le cirque, répondait à la volonté de s'appuyer sur des traces "ayant inspiré la mise en scène, plutôt que d'une retranscription vidéographique de l'œuvre afin de ne pas risquer l'imitation ${ }^{41}$ ». Derrière le rejet de l'imitation, ce qu'on voit apparaitre c'est bien la notion d'œuvre venant se constituer en pièce d'un répertoire.

Qu'il bâtisse des fondations pour édifier une nouvelle image de la discipline, ou qu'il permette au créateur d'entrer, à titre individuel, dans la lumière en reprenant une œuvre culte (monter son Sacre du Printemps, c'est un peu comme monter son Hamlet, une étape académique permettant d'obtenir ses lettres de noblesse), l'acte de reprendre a souvent à voir avec la légitimation par les pairs.

Le texte n'étant plus (seulement) au cœur du processus de création, la question du répertoire commence aussi à toucher la mise en scène, qui fait l'objet de nouveaux enjeux de conservation. Ce phénomène global qui touche les arts de la scène est à replacer dans un rapport étroit de notre société à la mémoire, ainsi que l'explique Emmanuel Wallon, professeur en sociologie de la culture : «Cette capacité de rétention de la collectivité doit compenser l'accélération sensible, brutale, des rythmes de la vie individuelle ${ }^{42}$.»

\section{Le besoin d'expérience}

Revenons à la question de Richard Schechner, car derrière cette interrogation se trouve également la suspicion d'une incapacité à l'oubli, de la part de cette génération, la nôtre, qui a vu grandir l'environnement digital, suspicion qui repose sur l'hypothèse que les nouvelles technologies captent des images du passé et les rendent accessibles, par là, omniprésentes. Mais cet environnement volatil, impalpable, immatériel, pourrait également expliquer cet impératif accru d'un besoin d'expérience, de passer par les corps, de sentir le temps vivant.

Si ce besoin d'expérience peut apparaître comme propre à une génération du «do it yourself ", cela renvoie aussi à une nécessité des arts vivants quant à la transmission de ses créations - les supports de notations et d'enregistrements ne sont pas suffisants, dans le sens qu'ils ne remplacent pas le vécu des corps, qu'ils figent alors que le corps joue de l'altération et des déplacements de cette matière - ainsi qu'à la réalité d'une génération dont les pères meurent les uns après les autres, sans qu'on ait eu à les tuer. Les disparitions de figures mythiques de la danse moderne et contemporaine, comme Pina Bausch et Merce Cunningham en 2009, du théâtre, comme Patrice Chéreau en 2013, et de la performance, comme Allan Kaprow en 2006, ont posé la question de la transmission et 
de la postérité de leurs œuvres et méthodes. Marina Abramovic réagit en multipliant les expériences de reprise de ses performances même avant la rétrospective au MoMA, crée maintenant un institut pour transmettre sa méthode, et a récemment joué sa mort dans le dernier spectacle de Bob Wilson, The Life and Death of Marina Abramović. Laure Fernandez, dans sa contribution à ce dossier ${ }^{43}$ rapporte la réflexion de Boris Charmatz et de Jérome Bel, suite au décès d'Odile Duboc et de Vincent Druguet, à propos de la nécessité de penser ce qu'ont laissé ces pères dans les corps. Si la danse a développé des outils formels de transmission (sources écrites, iconographiques, audio-visuelles...), Boris Charmatz propose de retrouver cette mémoire par l'improvisation, ou plutôt par l'«improvisation-archéologie », qu'il décrit non pas comme "un instantané posé sur la page vierge mais, au contraire, une archéologie au présent, une fouille qui réarticule le passé du corps à son écriture en temps réel ${ }^{44}$. "

Tuilant les mémoires, mémoires des corps, mémoires visuelles, mémoires de travail, rendant incontournables la présence des interprètes, mémoires construites des traces sur différents supports, on trouvera dans ce dossier de nombreux éclairages sur ces questions de transmission, d'appropriation, en danse, en théâtre, en cirque, pour la performance, d'un interprète à l'autre, en fonction des supports, mais aussi dans les techniques d'écriture singulières que cela développe (collage, assemblage, montage). Parlant de ces pratiques, au-delà de la question des intentions en amont et des critiques ou de la réception en aval - qui tous deux nous intéressent pour ce qu'ils disent de notre époque la reprise, agissant comme un combustible, se révèle être un biais intéressant pour aborder les questions de la création, de ses processus, de ses stratégies, de ses dramaturgies.

Ce besoin d'expérience pour les artistes, de traverser les choses en les éprouvant par le corps, en tissant un lien intime et charnel avec la mémoire, touche avec encore plus de force le public, qui, en accédant à une œuvre marquante de l'histoire du spectacle vivant, accède à un patrimoine oublié.

La culture de masse a donné accès à l'image des chefs-d'œuvre en la reproduisant sur de nouveaux supports virtuels ou produits dérivés. Etudiant le changement du statut de "l'œuvre d'art à l'époque de sa reproductibilité technique ${ }^{45}$ ", permise par l'imprimerie et la photographie, Walter Benjamin constate la déperdition de l' «aura", par la désincarnation du support de l'original. L'objet dans sa matérialité perd donc de son aura (l'expérience de l'objet original étant détruite par l'expérience de ses duplications), mais cette aura est augmentée par la fascination pour cette expérience, elle est ce qui précède l'objet. « La trace est l'apparition d'une proximité, quelque lointain que puisse être ce qui l'a laissée. L'aura est l'apparition d'un lointain, quelque proche que puisse être ce qui l'évoque. Avec la trace, nous nous emparons de la chose ; avec l'aura, c'est elle qui se rend maitresse de nous ${ }^{46}$.» En dupliquant l'œuvre, on attise l'envie de l'original, de pouvoir voir en vrai les peintures de Rafaël, pour dire que l'expérience a pris le pas sur l'image. Ainsi s'établit une sorte de safari culturel au fil duquel on va consommer du produit culturel et rechercher la sensation forte : toucher du doigt, voir, vivre le chef-d'oeuvre. On ne descend pas de sa voiture, mais on voit le lion passer tout près, de même, on voit la Joconde derrière sa vitre et la foule amassée, ou Einstein on the Beach ${ }^{47}$, mais presque trente ans ont passé. Etre en présence de l'œuvre mythique, dont on a tant de fois entendu parler. Vivre l'émotion esthétique. Et pouvoir dire : «j’y étais », « I was there ». 


\section{« En attendant la nouvelle Pina » : perspectives critiques de la reprise}

\section{La ligne de partage entre l'artistique et le culturel}

31 Si ce désir d'expérience est satisfait en partie, pour le moins en surface, par les signes qui ont permis de lui donner du crédit, la réception de la reprise - on entendra dans cette partie la reprise qui a trait aux grands noms, aux figures identifiées de l'histoire de l'art ou de l'histoire tout court, quelles qu'en soient les intentions, les postures et les formes tend à reconsidérer l'œuvre et la fonction des arts vivants comme une pratique sociale. Car l'expérience a pour mérite de créer une communauté de spectateurs, qui grossit de génération en génération, puisque ceux qui (re)voient rejoignent ceux qui avaient vu. La reprise apparaît comme le signe de l'absorption de l'art dans le culturel: elle signe l'entrée du spectacle dans le patrimoine collectif, l'œuvre ayant alors valeur comme élément de la culture commune et du lien social, plus que comme valeur artistique. Loin du débat et du dissensus, la reprise est le lieu du consensus, de l'accord immédiat, de la reconnaissance commune qui permet l'assimilation du «nouveau public» à une communauté qui sait ce qu'il faut voir. On attend de l'art qu'il réunisse, qu'il répare les fractures sociales, qu'il cicatrise.

La reprise est alors le point aveugle de la critique, car la chose vue n'est pas l'original, car la chose vue a comme rôle de témoigner d'un succès passé. Il est sur ce point significatif que les critiques de ces reprises soient presque unanimement enthousiastes, ou bien ne débattent que du degré de conformité entre l'objet présent et l'objet passé, dans une perspective souvent nostalgique, avec parfois un léger regret de la dimension commerciale, et réduisent finalement le discours à de la communication ${ }^{48}$.

On peut cependant se réjouir de cette accessibilité à une mémoire des arts vivants que permet la reprise, mémoire qui peut alors être transmise par d'autres réseaux, plus larges, plus médiatisés, et échapper à une disparition trop rapide des œuvres, qui empêche souvent une rencontre véritable avec le public. La reprise s'accompagne aussi d'un certain encouragement à la démocratisation culturelle.

\section{La réduction des risques}

La reprise dans son angle consensuel permet bien entendu de minimiser les risques pour les programmateurs ou directeurs de lieux, attirant des publics variés dans les salles de théâtre et s'asseyant sur la sécurité relative d'un succès passé. Ce phénomène est largement guidé et orienté par les stratégies marchandes qu'ont adoptées les institutions culturelles, avec lesquelles se débattent les financeurs publics et les lieux subventionnés.

Avec la reprise, la programmation culturelle se constitue en événement ou en logique événementielle. En s'adossant sur les grands noms du paysage artistique français et international, on entretient un star-system, une logique de vedettariat que le théâtre d'art avait banni. Les grands noms de la mise en scène sont mis en avant dans des " portraits », des « rétrospectives", des «focus », véritables temps forts de la programmation. Chaque année par exemple, le Festival d'Automne met en avant une figure importante, en 2013 Bob Wilson, l'année précédente Maguy Marin, desquels on voit plusieurs spectacles retraçant l'ensemble de leur parcours. Bien que l'arrêt sur le temps ou le retour en arrière 
proposé par ces focus propose ainsi une nouvelle lecture des œuvres de l'artiste, par leur simple mise en présence d'une part, et s'oppose d'autre part à l'injonction du nouveau qui a été le propre des politiques culturelles pendant plusieurs décennies (une «course épuisante à la création ${ }^{49}$ " selon Robert Cantarella), constituant une volonté d'aller à l'encontre de la spirale de la sur-production et de l'oubli - spirale bien connue qui va de pair avec la difficulté des compagnies à tourner - le principe s'apparente aussi à des stratégies marketing clairement identifiables : attirer le public vers des valeurs sûres, des spectacles ayant comme valeur ajoutée le fait d'être des «événements » identifiables et bénéficiant d'un écho médiatique important.

La reprise est-elle donc une nouvelle stratégie commerciale appliquée par les théâtres en temps de crise ? N'était-ce pas le cas de Knock qui remplissait les caisses de la compagnie de Louis Jouvet à chaque fois qu'elle approchait de la faillite? Pour autant, Emmanuel Wallon souligne les coûts engagés pour reprendre un spectacle d'envergure, mettant en jeu une nouvelle production, et relativise ainsi la dimension économique d'un tel choix de programmation. $\mathrm{Au}$ contraire, une analyse d'Isabelle Assassi ${ }^{50}$ comparant les programmations d'un $\mathrm{CDN}$, d'une scène nationale et d'un théâtre municipal au cours de la saison 2001-2002, citée par Daniel Urrutiaguer ${ }^{51}$ montre que les créations sont plus nombreuses quand les compagnies ont été à l'origine du choix du spectacle, et les reprises sont plus nombreuses quand la programmation provient des théatres : "Une orientation "stratégique", marquée par la recherche d'une péréquation des risques en prenant en compte les attentes du public, induit une large domination des reprises sur les créations ».

37 Ainsi, si la volonté de défendre la création a été pendant longtemps le fleuron des politiques culturelles françaises, il semble que les dernières décennies connaissent une inflexion vers la multiplicité des reprises, dans une optique de réduction des risques.

\section{«The Artist is present »}

Réduire les risques semble avoir comme corollaire l'identification de figures mythiques, reconnues et médiatisées. Le titre de l'exposition du MoMA «The Artist is present ${ }^{52}$ " exploite ce nouveau statut de l'artiste, tout autant que la performance de Marina Abramović, assise sur une chaise et fixant dans les yeux les spectateurs venu se mettre face à elle pendant de longues minutes sans ciller, huit heures par jour pendant trois mois. L'artiste présent apporte une valeur ajoutée à l'événement et devient «artiste entrepreneur en signature vivante ${ }^{53}$ ». Même absent, c'est encore sa présence, à travers d'autres corps que l'on va rechercher. C'est le corps de Lucinda Childs que l'on voit à travers celui de Kate Moran dans la reprise d'Einstein on the Beach en 2012, c'est l'âme de Bob Wilson que l'on voit à travers le décor et l'usage des lumières. On touche le voile du fantôme.

De même, l'artiste prend, selon Pascal Ory, une place nouvelle au sein d'une société désenchantée, devenant "nouveau saint ", «martyr ", "gourou » ou "prophète ${ }^{54}$ ", s'inscrivant dans une religion culturelle qui fait de l'œuvre d'art un objet sanctuarisé. Si le $20^{\text {ème }}$ siècle avait progressivement ébréché la statuette sacrée du génie créateur, il a fallu seulement quelques décennies pour y revenir. 


\section{Tautologie de la reprise}

40 L'accession de certains artistes de la contestation au rang de grands noms des arts vivants les expose dans leur image de figure, annulant ainsi leur rôle transgressif. La reprise bégaie alors au point de ne plus rien produire que ce borborygme. Elle n'ajoute pas plus qu'elle ne retire, elle annule. La critique principale de la reprise, questionnant la légitimité de cette pratique, repose sur le fait que le spectacle repris devient un objet empaillé, bien identifié et cerné, dépouillé de son intention, le mouvement de vie. Catherine Bouko et Giulio Boato ${ }^{55}$ questionnent cette dérive possible de la pratique: «Frédéric Maurin souligne le risque de dévitaliser l'œuvre en la réifiant et de la transformer en simulacre à partir du moment où la valeur d'échange supplante la valeur d'usage ${ }^{56}$. Ce qui apparaît de façon accrue avec la reprise des œuvres subversives telles que celles de Jan Fabre, reposant sur une physicalité forte et sur une radicalité des actions. Reprendre ce qui a choqué se heurte au danger de rendre le subversif convenu et de mesurer, par l'écart, que cela ne choque plus - comme les corps nus de Dionysus in 69, véritable libération des mœurs à l'époque, qui sont aujourd'hui devenus monnaie courante (ou presque). La reprise des avant-gardes des années 60 et 70 n'y déroge pas. Reprendre de tels spectacles les fige dans leur forme d'autrefois, devenant une entrave au déploiement du sens. Marie Quiblier rappelle ce que Larys Frogier dit de l'obscène : « l'obscène, c'est-à-dire l'en-deça de la scène, est ce qui annule le rapport scénique habituel d'une mise à distance suffisante du spectateur pour qu'il jouisse aisément de son statut de voyeur ${ }^{57}$ ».

\section{Avant de partir}

Dix ans après une mise en scène à l'Odéon dont il était alors directeur, George Lavaudant remonte La Mort de Danton ${ }^{58}$ de Büchner. À peu de rôles près, la distribution est la même et le metteur en scène s'en explique : "C'est comme si c'était les fantômes de la pièce qui revenaient nous la raconter à nouveau ${ }^{59} "$, et le rapproche du propos dramaturgique de l'œuvre : «ce sont tous des morts, justement, plus ou moins en sursis ». Ceux qui seront tôt ou tard conduits à la guillotine, opposants à Robespierre, pris dans cette folle mécanique de la Terreur, ne trouvent plus les mots. Danton abdique, jusqu'au sursaut final, et se demande: est-ce l'histoire qui fait les hommes ou les hommes qui font l'histoire ? La pièce, créée en 2002 juste avant la fermeture de l'Odéon pour travaux, s'achevait par l'ouverture des portes de fond de scène sur les grilles du jardin du Luxembourg et la mise face à face du public et des passants, foule anonyme venant regarder curieusement le spectacle de l'extérieur. Par hasard, la représentation du spectacle s'est inscrite dans le contexte des élections présidentielles et du passage de Jean-Marie Le Pen au second tour, ce qui donnait une résonnance politique particulière au spectacle. En 2012, la reprise s'inscrit également dans le contexte des élections et Lavaudant de noter : « Mais, encore une fois, ce n'est pas la raison pour laquelle on monte une pièce, en misant sur une actualité immédiate ${ }^{60}$. "

Cette dernière phrase pousse à s'interroger et pointe une ambivalence fondamentale de la reprise: elle contient en elle un risque d'érosion de ses affirmations esthétiques, d'altération de son sens, voire de sa fonction critique, et porte en même temps en elle, de façon aléatoire, accidentelle, ou intentionnelle, cette force de questionnement du présent 
par la réitération du passé. Ainsi, la reprise peut avoir la vertu d'un surgissement, celle d'un passé inédit, subversif, qui pénètre les grilles de notre temps, « saturé d'à-présent ${ }^{61}$ » comme le dit Benjamin, trop myope pour se penser lui-même. Les fantômes de Büchner ne reviendraient-ils pas reposer au présent la question de cette puissance d'agir?

Les avatars de la reprise, qu'ils agissent de façon respectueuse ou iconoclaste, fragmentaire ou totalisante, allusive ou frontale, réitèrent ce questionnement et invitent à reconsidérer des catégories traditionnelles et des fractures classiques entre innovation et répétition du même; entre avant-garde et académisme ; entre crise et progrès; entre artistes, programmateurs et critiques (public et critique médiatique compris); entre annulation et puissance d'action, entre idéologie (comment l'œuvre reprend et répète les éléments idéologiques de son époque) et utopie (comment elle s'en arrache et produit un réagencement à l'intérieur de la reprise) selon les termes de Jameson ${ }^{62}$. C'est à cet endroit de l'impossibilité de penser la reprise sans les relations qui la font exister, qu'il nous semble que ce dossier invite - dimension critique à laquelle les articles, entretiens et témoignages rassemblés dans ce dossier nous semblent pouvoir apporter quelque matière à réflexion.

\section{NOTES}

1. Jean De La Bruyère, Les Caractères ou les mœurs de ce siècle, éd. Estienne Michallet, 1696, Des ouvrages de l'esprit, p. 7.

2. Jérome Bel, Véronique Doisneau, solo pour la danseuse éponyme pour l'Opéra de Paris, 2004. Des extraits vidéo sont consultables à ce lien: http://artforum.com/video/ id=26359\&mode=large\&page_id=9 . Coproduction de l'Opéra National de Paris, Telmondis, en association avec France 2, avec la participation de Mezzo, sous la direction de Jérome Bel et Pierre Dupouey.

3. Ea Sola, interviewée par Jean Pierre Han (voir Entretien réalisé par Jean-Pierre Han, Revue Frictions, $\mathrm{n}^{\circ}$ 22, Hiver 2013-2104, pp.61- 64.) à propos de la reprise de Sécheresse et pluie (recréation en 2011, création en 1995), dit avoir fait un travail de "mémoire », autour de la guerre du Vietnam, alors que sa première création était un travail de "souvenir", elle retraverse, avec d'autres interprètes, cette mémoire du conflit.

4. Elle peut être une forme d'emprunt libre, comme Boris Charmatz puisant dans l'histoire de la danse pour lui faire des pieds de nez, une forme de "recyclage» de sa propre matière chorégraphique réexploitée et retransmise à travers divers spectacles (Xavier Le Roy), une « re| action ", les gens d'Uterpan utilisant ce nom pour désigner le re-questionnement du lieu et du contexte social et politique dans lequel ils reprennent leur dispositif.

5. «La DRAC offre la possibilité d'une aide à la reprise d'un spectacle « qui a bénéficié d'une aide de l'État et qui a connu par la suite une interruption d'exploitation d'au moins une saison. » in Daniel Urrutiaguer, "Reprises de spectacles et valorisation de la production artistique ", Agôn [En ligne], Dossiers, $\mathrm{N}^{\circ} 6$ : La Reprise, mis à jour le: 17/02/2014, URL: http:// journals.openedition.org/agon/2738.

6. Voir l'article de Marie Quiblier, «Le Sacre du printemps - Mise en perspective des relectures d'Yvonne Rainer et de Xavier Le Roy : pour un détournement des pratiques et des regards ", Agôn 
[En ligne], $\mathrm{N}^{\circ} 6$ : La Reprise, Dossiers, mis à jour le: 17/02/2014, URL: http:// journals.openedition.org/agon/2782.

7. Intervention au colloque à l'occasion du centenaire du Sacre du printemps organisé par le Kaiitheater et Charleroi/Danses le 21 novembre 2013 à La Raffinerie, Bruxelles. Cf. L'intervention de Dominique Brun, disponible dans ce dossier « Reprise » : 1913//2013 : Le Sacre du Printemps.

8. Frédéric Pouillaude, Le désoeuvrement chorégraphique, étude sur la notion d'œuvre en danse. Paris, Vrin, 2009.

9. Sören Kierkegaard, Nelly Viallaneix (trad.), La Reprise, Paris : Ed. Flammarion, Coll. Grand Format, 1990. pp. 65-66.

10. Cette tradition perdure, entreprise à la fois gigantesque et tatillonne, et fêtera l'année prochaine son bicentenaire.

11. Slavoj Zizek, « Lenin's legacy », From: London Review of Books, vol. 24 No. 14, 25 July 2002.

12. On ne développera pas ici les questionnements sur le plagiat, forme qu'on situera à la lisière du spectre de ce que recouvre la reprise, en tout cas dans le débat moral qui lui est associé. Voir par exemple pour une problématisation de cette notion l'ouvrage de Michel Schneider Voleur de mots, essai sur le plagiat, la psychanalyse et la pensée, Paris, Gallimard, 1985. Dans le cadre de ce dossier, on renvoie à l'article de Roland Huesca, "Nijinski chorégraphe ", http:// journals.openedition.org/agon/2730, qui aborde notamment la question sous l'angle juridique du droit d'auteur, à travers l'exemple du Sacre du Printemps. On ne peut pas passer sous silence la polémique qu'a déclenché le plagiat de Rosas danst Rosas, chorégraphie d'Anne Teresa de Keersmaeker (1983) par la chanteuse Beyoncé pour sa chanson «Countdown" (voir la vidéo suivante : http://www.youtube.com/watch?v=3HaWxhbhH4c), qui a abouti, paradoxalement peut-être, à l'ouverture d'un site, à l'initiative de la chorégraphe flamande, pour apprendre en ligne la chorégraphie Re: Rosas (http://www.rosasdanstrosas.be/accueil) .

13. Sigmund Freud, "Über den psychichen Mechanismus hysterischer Phänomene", in: Gesammelte Werke in achtzehn Bändern mit einem Nachtragsband. Frankfurt a. M., 1987, p. 193, cité par Inke Arms, History will repeat itself, Strategies of re-enactment in contemporary (media) art and performance, Exhibition at KW Institute for contemporary art in Berlin, 18/11/2007 - 13/01/2008, p. 39.

14. Inke Arms, Op. cit.

15. Richard Schechner, p. 36.

16. Ibidem.

17. "All representational practice, and indeed all communicative behavior, is composed in reiteration, is engaged in citation, is already a practice of reenactment, or what Richard Schechner has termed "restored" or "twice-behaved behavior."” in Rebecca Schneider, Performing Remains. New York : Routledge, 2011, p. 10.

18. Voir l'article de Roland Barthes «Théorie du texte", publié dans l'Encyclopédie Universalis en 1974.

19. Julia Kristeva, Séméiotiké, Recherches pour une sémanalyse, Seuil, Paris, 1969.

20. Notion formalisée par Gérard Genette, dans Palimpsestes, la littérature au second degré, Seuil, Paris, 1982.

21. Jacques Derrida, La carte postale, de Socrate à Freud et au-delà, Paris, Flammarion, 1980, pp. 13-14.

22. Sans développer dans le cadre de cette introduction, on renvoie aux ouvrages des tenants de l'histoire culturelle tels que Roger Chartier, Pascal Ory, Dominique Kalifa, qui analysant les questions de mémoire et de patrimoine ont développé la construction épistémologique de cette histoire.

23. "Disons pour faire bref que l'histoire, dans sa forme traditionnelle, entreprenait de "mémoriser » les monuments du passé, de les transformer en documents et de faire parler ces traces qui, par elles-mêmes, souvent ne sont point verbales, ou disent en silence, autre chose que 
ce qu'elles disent ; de nos jours, l'histoire, c'est ce qui transforme les documents en monuments, et qui, là où on déchiffrait des traces laissées par les hommes, là où on essayait de reconnaître en creux ce qu'ils avaient été, déploie une masse d'éléments qu'il s'agit d'isoler, de grouper, de rendre pertinents, de mettre en relations, de constituer ensembles. Il était un temps où l'archéologie, comme discipline des monuments muets, des traces inertes, des objets sans contexte et des choses laissées par le passé, tendait à l'histoire et ne prenait sens que par la restitution d'un discours historique; on pourrait dire, en jouant un peu sur les mots, que l'histoire, de nos jours, tend à l'archéologie, - à la description intrinsèque du monument. ", Michel Foucault, Archéologie du savoir, Archéologie du savoir, Paris, Gallimard, «Bibliothèque des sciences humaines ", 1969, rééditions 1992, p. 14-14.

24. Arnauld Pierre, Futur Antérieur. Art contemporain et rétrocipation, Paris, M19, 2012, p .9. Cité par Laure Fernandez, Laure Fernandez, " "Travailler contre des fantômes »", Agôn [En ligne], Dossiers, $N^{\circ} 6$ : La Reprise, mis à jour le : 21/02/2014, URL : http://journals.openedition.org/ agon/2799.

25. Voir l'entretien réalisé avec Franck Apertet, « Faire jaillir le cadre », Franck Apertet, « Faire jaillir le cadre », Agôn [En ligne], Dossiers, $\mathrm{N}^{\circ} 6:$ La Reprise, mis à jour le : 14/02/2014, URL : http://journals.openedition.org/agon/2779.

26. Isabelle Launay, "Poétiques de la citation en danse - ... d'un Faune (éclats) du Quatuor Albrecht-Knust, avant-après 2000 ", in Isabelle Launay et Sylviane Pagès [dir.] Mémoires et histoire en danse, Mobiles $n^{\circ}$ 2, coll. Arts 8, Paris, L'Harmattan, 2010. p. 25.

27. Dans son travail sur l'histoire du temps présent, Henry Rousso se livre à une historiographie de la contemporanéité et explique que pour aujourd'hui « la contemporanéité ne définit pas un moment figé du temps mais un mouvement en cours ». Voir Henry Rousso, La dernière catastrophe. L'histoire, le présent et le contemporain, Paris, Gallimard, « NRF Essais », 2003. p. 175.

28. "So the question I would ask back to Ariane, and people who are younger than me: why are you talking to me? What has happened ? of course, on my individual ego level, I'm glad you're talking to me. But historically, structurally..." Christian Biet et Richard Schechner , "Reprendre les performances de l'Avant-Garde ", Agôn [En ligne], Dossiers, $\mathrm{N}^{\circ} 6$ : La Reprise, mis à jour le : 14/02/2014, URL : http://journals.openedition.org/agon/2778.

29. "Exit Thirties, Enter Sixties" in Public Domain: Essays on the Theatre. Indianapolis : BobbsMerrill, 1969.

30. Pier Paolo Pasolini, Les lettres luthériennes, Paris, Editions du Seuil, 2002.

31. C'est ce que déclare Peggy Phelan, in Unmarked: The Politics of Performance. London, New York : Routledge, 1993. p. 147.

32. Marina Abramović crée un institut portant son nom au sein duquel seront transmises ses «méthodes pour augmenter l'attention au présent et explorer les frontières entre corps et esprit », https://mai.art.

33. Ces questions sont développées dans l'article d'Alice Carré, «Les reprises à l'affiche : peur de l'oubli ou rentabilité ? ", Revue Frictions, n²2, Hiver 2013-2014, p. 58. Nous invitons par ailleurs à consulter ce numéro de la revue Frictions, qui regroupe plusieurs articles traitant de la question de la reprise : un entretien avec la chorégraphe Ea Sola, cité précédemment, et un article de Robert Cantarella.

34. Roland Huesca, « Nijinski chorégraphe ", Agôn [En ligne], $N^{\circ} 6:$ La Reprise, Dossiers, mis à jour le : 14/02/2014, URL : http://journals.openedition.org/agon/2730.

35. Les Médusés, parcours chorégraphique de Damien Jalet, présenté au Louvre, 2013.

36. Le spectacle, nommé presque ironiquement Rétrospective, part du constat de l'impossibilité de faire la propre généalogie de son œuvre. Rien ne peut exister de manière figée, tout doit être transmis à d'autres corps, métamorphosé et détourné, remis en jeu. Si la danse se rapproche du musée, c'est en effet que la question de construire la mémoire de sa propre discipline se pose de façon nouvelle. Voir le dossier artistique du présent dossier lui étant consacré. 
37. Marie Fonte, "Écrire le cirque ", Agôn [En ligne], Dossiers, $N^{\circ} 6:$ La Reprise, mis à jour le : 20/02/2014, URL : http://journals.openedition.org/agon/2787.

38. Fugues, mise en scène de Yoann Bourgeois, créé en 2008 et repris en 2011.

39. In Vitro, spectacle créé par le collectif Archaos en 1999. La pièce a été recréée "à l'identique » par les élèves de l'école du cirque de Rio de Janeiro en 2009, puis recréée également en 2009 par Archaos dans une nouvelle version appelée In Vitro 09.

40. Raquel de Andrade et Guy Carrara, « Points de vue sur la notion de répertoire dans les arts du cirque » (10 novembre 2010), in "In Vitro 09 », Pièce (dé)montée, n 117, novembre 2010, URL : http://crdp.ac-paris.fr/piece-demontee/pdf/in-vitro-09_total.pdf., cité par Barbara MétaisChastanier, «Écriture(s) du cirque : une dramaturgie? », Agôn, Enquêtes, Dramaturgie des arts de la scène, mis en ligne le 18 octobre 2012. Bientôt en ligne à cette URL: http:// journals.openedition.org/agon/2308.

41. Id.

42. Emmanuel Wallon, « « Un avertissement contre l'oubli » ", Agôn [En ligne], Dossiers, $\mathrm{N}^{\circ} 6$ : La Reprise, mis à jour le : 20/02/2014, URL : http://journals.openedition.org/agon/2797.

43. Laure Fernandez, " "Travailler contre des fantômes " ", Agôn [En ligne], Dossiers, $\mathrm{N}^{\circ} 6: \mathrm{La}$ Reprise, mis à jour le : 21/02/2014, URL : http://journals.openedition.org/agon/2799.

44. Jérôme Bel et Boris Charmatz, Emails 2009-2010, Dijon, Les Presses du Réel, 2013, pp. 178-179.

45. Walter Benjamin, L'œuvre d'art à l'époque de sa reproductibilité technique, Paris, Allia, 2003.

46. Walter Benjamin, Paris, capitale du XXIe siècle, Le livre des passages, Paris, Editions du Cerf, 1989, p. 464

47. Nous renvoyons ici à l'enquête, Einstein on the beach, 1976 // 2012, dirigée par Barbara MétaisChastanier et Edwige Perrot, http://journals.openedition.org/agon/2166.

48. Voir Diane Scott, Avignon 2009, Carnets critiques, Paris, L'Harmattan, 2009, p. 19-21.

49. Robert Cantarella, «Refaire, répéter, recommencer, essayer pareil », Revue Frictions $n^{\circ} 22$, Hiver 2013-2014, p. 56.

50. Isabelle, Assassi, "The Programming Strategies and Relationships of Theatres: An Analysis Based on the French Experience", International Journal of Arts Management, vol. 9, $\mathrm{n}^{\circ}$ 3, 2007, Spring, p. 57.

51. Daniel Urrutiaguer, « Reprises de spectacles et valorisation de la production artistique », Agôn [En ligne], Dossiers, $\mathrm{N}^{\circ} 6:$ La Reprise, Reprendre pour faire bouger les cadres, mis à jour le : 17/02/2014, URL : http://journals.openedition.org/agon/2738.

52. Rétrospective Marina Abramović, « The Artist is Present » au MoMA de New York, 2010.

53. Isabelle Barbéris et Martial Poirson, L'Economie du spectacle vivant, Paris, Que sais-je ?, Presses Universitaires de France, Juin 2013, p. 110.

54. Pascal Ory, "Vous avez dit "Chef-d'œuvre" ?», Introduction à une histoire de la religion culturelle, Paris, Editions Jean-Michel Place, 2013, p. 18.

55. Voir Giulio Boato et Catherine Bouko, "Jan Fabre au risque de la reprise ", Agôn [En ligne], Dossiers, $N^{\circ} 6$ : La Reprise, mis à jour le: 19/02/2014, URL : http://journals.openedition.org/ agon/2741.

56. Frédéric Maurin «Sous la cendre, les braises : actualité et usages des années 1960-1970", Théâtre/Public, janvier-mars 2012, n 203, p. 17, cité par Catherine Bouko et Giulio Boato, art. cit.

57. Larys Frogier, "S'exposer, quand l'artiste dévisage la pornographie», Sous titrée X: la pornographie entre image et propos, cat. d'expo, Rennes, P.U.R, 2001 (Collection Métiers de l'exposition). Cité par Marie Quiblier, « Le Sacre du printemps - Mise en perspective des relectures d'Yvonne Rainer et de Xavier Le Roy : pour un détournement des pratiques et des regards ", Agôn [En ligne], $\mathrm{N}^{\circ} 6$ : La Reprise, Dossiers, mis à jour le: 17/02/2014, URL : http:// journals.openedition.org/agon/2782.

58. La Mort de Danton de Georg Büchner, mise en scène Georges Lavaudant, créé en 2002, Théâtre de L'Odéon, reprise en 2012 à la MC93. 
59. Entretien entre Georges Lavaudant et Daniel Loayza, www.mc93.com.

60. Id.

61. Walter Benjamin, Sur le concept d'histoire, Thèse XIV, Paris, Petite Bibliothèque Payot, Juin 2013.

62. Fredric Jameson, Le Postmodernisme ou la logique culturelle du capitalisme tardif, Paris, ENSBA Editeur, coll. « D'art en questions », 2007.

INDEX

Mots-clés : Abramovic (Marina), archéologie, Barthes (Roland), Bausch (Pina), Benjamin (Walter), cirque, citation, critique, culture, danse, histoire, Doisneau (Véronique), monument, Bel (Jérôme), nostalgie, patrimoine, performance, reconstitution, recréation, reenactment, reprise, théâtre, Schechner (Richard) 